\title{
Mobilité Géographique Intraorganisationnelle au Togo : Une Etude Exploratoire des Perceptions et des Vécus
}

\author{
Pazambadi Kazimna, \\ Enseignant-chercheur à l'Université de Lomé, Togo
}

Doi:10.19044/esj.2020.v16n17p167 URL:http://dx.doi.org/10.19044/esj.2020.v16n17p167

\section{Résumé}

L'objectif de la présente étude est d'analyser les perceptions et le vécu de la mobilité géographique intra-organisationnelle des travailleurs au Togo. Pour ce faire un questionnaire construit a été administré à 45 travailleurssalariés togolais issus des villes et des zones rurales. Ces travailleurs ont fait l'objet d'une mobilité géographique au moins. Les résultats obtenus montrent qu'il existe des perceptions de freins à la mobilité des salariés togolais. Ces freins sont liés entre autres à l'âge, à la situation familiale, à l'absence de politique de mobilité, et aux difficultés d'accès à certaines zones. Ce qui entraine, dans les vécus de certains travailleurs, le stress et le sentiment d'injustice. Toutefois, l'étude révèle également que la mobilité géographique, qui est un outil de management des ressources humaines et un facteur de réduction de la disparité entre villes, préfectures et régions, présente aussi des opportunités de développement professionnel (promotion).

Mots-clés : Mobilité géographique, perceptions, vécu, intra-organisationnelle, travailleurs 


\title{
Geographical Intraorganizational Mobility in Togo: An Exploratory Study of Perceptions and Real-Life Experiences
}

\author{
Pazambadi Kazimna, \\ Enseignant-chercheur à l'Université de Lomé, Togo
}

\begin{abstract}
The objective of this study is to analyze the perceptions and real-life experience of intra-organizational geographic mobility of workers in Togo. To do this, a constructed questionnaire was administered to 45 Togolese workers and salaried workers from cities and rural areas. These workers have been subject to at least geographic mobility. The results obtained show that there are perceptions of obstacles to the mobility of Togolese employees. These obstacles are linked, among other things, to age, family situation, lack of a mobility policy, difficulties in accessing certain areas. This leads to the stress and the feeling of injustice experienced by certain workers surveyed. However, the study also reveals that geographic mobility, which is a human resource management tool and a factor in reducing the disparity between cities, prefectures and regions, also presents opportunities for professional development (promotion).
\end{abstract}

Keywords: Geographic mobility, perceptions, real-life experience, intraorganizational, workers

\section{Introduction}

Les sociétés du sud sont entrées de plein fouet dans une économie de marché de plus en plus mondialisée, sans avoir été partie prenante de son organisation (Mokounkolo, 2019, p. 7). Ainsi, dans cet environnement, où la mondialisation a pris le pas, l'objectif de toute entreprise, de toute organisation est la course à la compétitivité en changeant ou en améliorant les techniques de production, de prestation et de gestion des ressources humaines. Les Etats sont ainsi appelés à jouer un rôle déterminant et prioritaire dans ce contexte. A cet effet, dans le prolongement des objectifs du Millénaire pour le développement (OMD), les chefs d'Etats et de gouvernement et hauts représentants, réunis au siège de l'Organisation des Nations Unies à New York du 25 au 27 septembre 2015, ont adopté un nouveau programme pour le développement durable à caractère universel, applicable dans son intégralité à 
l'horizon 2030 dans ses trois dimensions (économique, sociale et environnementale) d'une manière qui soit équilibrée et intégrée.

Dans ce contexte, les managers des organisations éprouvent des difficultés face à la complexe mission de pourvoir aux besoins de chaque service les ressources, notamment humaines, nécessaires en vue de l'atteinte des objectifs fixés, la performance escomptée et de réduire les inégalités. Aussi, la mobilité professionnelle apparaît-elle comme un outil important pour la gestion efficace et efficiente des ressources humaines des entreprises. La mobilité professionnelle est définie comme « une transition d'emploi interne ou externe, initiée par le travailleur ou imposée par l'organisation » (Hellemans et Equeter, 2016, P. 299).

Classiquement, la mobilité professionnelle est catégorisée comme inter-organisationnelle (externe) ou intra-organisationnelle (interne), verticale (hiérarchique), horizontale (fonctionnelle) ou radicale (rapprochement vers le centre de prise de décisions), définitive ou provisoire, nationale ou internationale, initiée par le travailleur (volontaire, choisie) ou imposée par l'organisation (subie) (Hellemans, Equeter, 2016).

Les sociétés du sud doivent en particulier, bien plus que la plupart de celles du Nord, relever de nombreux défis liés au travail pour assurer leur développement économique et social, et satisfaire les besoins de sécurité de leurs populations dans différents domaines (Mokounkolo, 2019). La mobilité géographique s'impose alors comme un outil incontournable d'optimisation des ressources humaines (Maclouf, Wierzbicki, 2008) publiques et privées dans ce contexte où la concentration des compétences et de la population s'observe plus dans certaines zones que d'autres. Pourtant, l'émergence des pôles de développement et de croissance dans les régions nécessite la mise sur pied d'un potentiel minimum de ressources humaines. En outre, dans le but de rendre viable la dynamique de la décentralisation, il importe de renforcer non seulement les capacités institutionnelles et organisationnelles des collectivités locales mais aussi et surtout des ressources humaines. Ces défis sollicitent en particulier la capacité des organisations à développer des outils pour améliorer non seulement l'employabilité et l'insertion professionnelle des salariés, mais aussi à appliquer un management des ressources humaines (Mokounkolo, 2019) susceptible de répondre à l'impératif majeur de la mobilité géographique. Les hommes sont ainsi appelés à jouer un rôle déterminant et prioritaire dans ce contexte. Ce qui pose indirectement le problème de la mobilité géographique des Ressources Humaines. La mobilité géographique, qualifiée d'instrumentale, est le principal moyen d'assurer l'adéquation entre les besoins et les ressources. Elle peut s'entendre donc comme le passage d'une zone à une autre. Il s'agit dans le cas de cette étude, d'une frontière administrative (ville, préfecture, région). 
De toute évidence, afin d'améliorer les pratiques de mobilité des entreprises, il faudrait prioritairement que des études prospectives puissent baliser la voie à la politique de mobilité géographique. A cet effet, dans le monde, plusieurs études se sont intéressées à la mobilité géographique des travailleurs. Ainsi, dans la perspective de développer une mesure capable d'attirer les travailleurs qualifiés dans les régions périphériques québécoises, Deschênes, Aguir, Beaudry et Laflamme (2018) pensent qu'il faut mettre en évidence un modèle de l'attraction régionale prenant en compte trois dimensions : l'environnement économique, la qualité de vie et la culture régionale. Des études ont montré que l'insertion professionnelle des jeunes entretient une relation significativement positive avec la mobilité géographique, si l'on contrôle les déterminants de l'accès à l'emploi comme le niveau de diplôme, le sexe et l'âge (Dumartin, 1995, Bernela, 2017). D'autres études montrent également qu'il existe un lien entre l'insertion professionnelle des femmes et leur mobilité spatiale, avec comme variable intermédiaire, l'accessibilité géographique des emplois (Vandersmissen, Villeneuve et Thériault, 2001).

Par ailleurs, la mobilité géographique et la promotion professionnelle des salariés sont étroitement liées. Etre promu implique souvent un déplacement géographique. Inversement, changer de lieu de résidence favorise l'avancement (Brutel, Jegou \& Rieu, 2000). Une caractérisation par type d'espace (« grand urbain », " petit urbain » et « espace à dominante rurale ») permet de préciser davantage que la mobilité géographique est toujours favorable à la promotion professionnelle (Brutel, Jegou \& Rieu, op.cit).

Enfin, des études montrent une interdépendance entre la carrière familiale et la mobilité géographique des couples (Bonnet, Collet et Maurines, 2006). Les couples effectuent des ajustements et des négociations afin de concilier la vie conjugale et familiale et la carrière professionnelle de chacun des conjoints.

Cependant, au Togo peu d'études se sont intéressées aux systèmes organisationnels des entreprises. Selon ces études, les entreprises publiques togolaises sont caractérisées par une mauvaise organisation, une faible coordination et une absence de culture du sens de devoir (Chitou, 2013). De plus, en dépit des progrès notables réalisés pour le développement inclusif au Togo, des défis importants persistent en termes d'inégalités sociales et spatiales, de faiblesse du système productif national, de ressources humaines et de gouvernance (République togolaise, 2018).

Par ailleurs, les théories de l'action raisonnée (Ajzen, 1991) semblent potentiellement plausibles pour mieux expliquer les attitudes des individus dans le contexte de la mobilité géographique. En effet, l'acceptation d'une proposition de mobilité renvoie à une intention. L'intention est également fondée sur une double conditionnalité : d'une part, l'espérance d'un gain ; 
d'autre part, la volonté de se conformer à une norme, un principe qui renvoie à une pression sociale, et correspond à une dimension du contrat psychologique. La dimension du contrat psychologique centralise la relation d'emploi autour d'un équilibre garanti par le respect des obligations réciproques entre employeur et employé (Rousseau, 1989) ; Ce qui permet de comprendre les conséquences néfastes de la mobilité géographique en terme de violation de contrat par l'employeur. La disposition envers la mobilité correspond alors à un état mental d'ouverture à un comportement et est censée contribuer à l'intention (la perception des fins et des moyens) de mobilité (Mignonac, 2004).

En somme, il faut noter que différents travaux dans la littérature internationale ont établi d'une part, le lien entre la mobilité géographique et l'insertion professionnelle, la promotion professionnelle, la carrière familiale des couples et d'autre part, montré leur inter influence. Au Togo les études n'ont montré que les spécificités des systèmes organisationnels et les représentations du secteur privé par les travailleurs.

C'est pourquoi, la présente étude exploratoire se fixe pour objectif d'analyser les perceptions et les vécus de la mobilité géographique intraorganisationnelle au Togo.

Par ailleurs, l'évolution des carrières et les opportunités de poursuivre des études supérieures sont souvent inexistantes ou difficiles à trouver surtout dans les zones rurales en Afrique (Adededji \& Olaniyan, 2011). Au Togo, les études montrent que les travailleurs (secteurs privé et public) ont une représentation mitigée de leur organisation en matière de gestion des ressources humaines (Pari, 2008; Kazimna, 2012). Se référant à cette littérature qui montre les disparités et les inégalités en Afrique en général et au Togo en particulier, on peut déduire que les travailleurs n'auront pas les mêmes attitudes face à l'affectation. Ainsi, la présente étude part de l'hypothèse selon laquelle la mobilité géographique des salariés au Togo est perçue et vécue différemment par les travailleurs affectés.

La présente étude s'articule en deux points: le premier point présente le cadre méthodologique (1) et le deuxième point est relatif aux résultats obtenus et leur discussion (2).

\section{Méthodes}

\subsection{Participants et technique de collecte des données}

La population d'étude est constituée des travailleurs du secteur formel du Togo. Ces travailleurs viennent des organisations qui sont implantées au moins dans deux villes ou régions ou préfectures (localités) du Togo. Cependant, il n'existe pas de base de données permettant d'identifier cette population en vue de recueillir leurs opinions permettant de réaliser une étude. Ainsi, la technique pertinente permettant le recrutement des participants paraît 
celle de «boule de neige ». Elle est utilisée dans des conditions où la population d'étude est cachée, difficile à trouver ou à atteindre à cause de leur relative rareté ou en l'absence d'une base de sondage appropriée (Johnston \& Sabin, 2010 ; Frank \& Snijders, 1994). Elle consiste à identifier des répondants qui doivent recommander à leur tour un nombre illimité de pairs.

Cette technique a consisté, dans le cadre de cette étude, à identifier en premier lieu un travailleur ayant été affecté d'une zone à une autre à l'intérieur d'une même organisation (intra-organisationnelle), il y a au moins un an (pour éviter l'effet direct des émotions). Ce participant ainsi identifié renseigne un questionnaire en ligne. Il envoie ensuite le lien du questionnaire à d'autres collègues qui ont été une fois affectés. De la même manière, ces collègues contactent d'autres répondants. Ainsi, l'effectif des participants obtenu s'élève à 45 travailleur-salariés, dont l'âge varie entre 23 et 56 ans. Les enquêtés recrutés venaient des banques, de la caisse Nationale de sécurité Sociale, de Togo-télécom, de Moov-Togo, de la Poste du Togo et des Assurances.

\subsection{Passation}

Les principales variables prises en compte dans la collecte des données sont relatives à la mobilité géographique, aux perceptions et au vécu. La mobilité géographique est le passage d'une zone à une autre dans une même organisation (entreprise). Il s'agit d'une frontière administrative (villes, préfectures, régions). La perception est l'image (ou la représentation) que se fait un travailleur de la mobilité géographique. Le vécu est l'ensemble des influences subies liés au phénomène d'affectation. La littérature met en évidence le fait que la mobilité géographique confronte le travailleur à des différentes situations pouvant perturber ses habitudes ou non. Le travailleur doit alors effectuer un travail de réorganisation permettant plus ou moins bien selon les cas une adaptions à la situation.

Pour la collecte d'informations sur les variables susmentionnées, un questionnaire a été élaboré. Il est structuré comme suit:

- quatre items permettant de cerner les caractéristiques de la population ;

- cinq items portant sur l'affectation (mobilité géographique), les préférences de localités de travail, la politique en matière de mobilité géographique, les perceptions;

- un item relatif au vécu des situations de mobilité géographique (affectation); et

- un item de proposition de solutions pour améliorer la gestion de la mobilité géographique au Togo.

Pour l'analyse des données, il a été fait recours aux méthodes quantitative et qualitative. En effet, il a été procédé à des analyses quantitatives avec des méthodes statistiques afin de calculer les fréquences et les 
pourcentages. L'analyse logico-sémantique a permis de compléter la méthode quantitative, pour faire ressortir le sens manifeste des réponses recueillies sur les questions ouvertes relatives aux vécus et suggestions d'améliorations de la mobilité géographique. Les paragraphes qui suivent se rapportent à la présentation et à la discussion des résultats.

\section{Résultats et discussion}

\subsection{Résultats}

L'analyse des données recueillies a permis d'obtenir les résultats suivants répartis en quatre points : (i) les caractéristiques des enquêtés ; (ii) les perceptions de la mobilité géographique; (iii) le vécu de la mobilité géographique et (iv) les propositions de solutions.

\section{(i) les caractéristiques des enquêtés}

Les salariés enquêtés avaient l'âge compris entre 23 et 56 ans et sont composés de 59,07 \% d'hommes, contre 40, $03 \%$ de femmes.

Fréquence des enquêtés selon le nombre d'affectations suivi

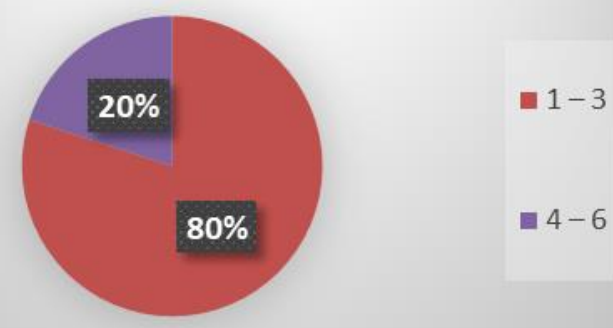

Graphique 1 : Répartition des enquêtés selon le nombre d'affectations

D'après le graphique1, 20\% des enquêtés ont été affectés au moins quatre fois (changement de plus de quatre localités) durant leur carrière.

(ii) Les perceptions de la mobilité géographique

Fréquence(\%) des enquêtés selon leur satisfaction du nouveau lieu d'affectation

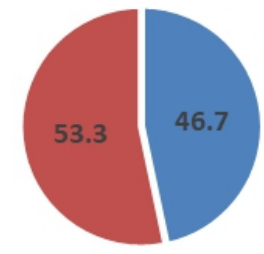

Graphique 2 : Répartition des enquêtés selon leur satisfaction liée à l'affectation 
Ce graphique 2 indique que la majorité des enquêtés, soit 53, 3\% ne sont pas satisfaits de leur nouveau lieu de travail après leur affectation.
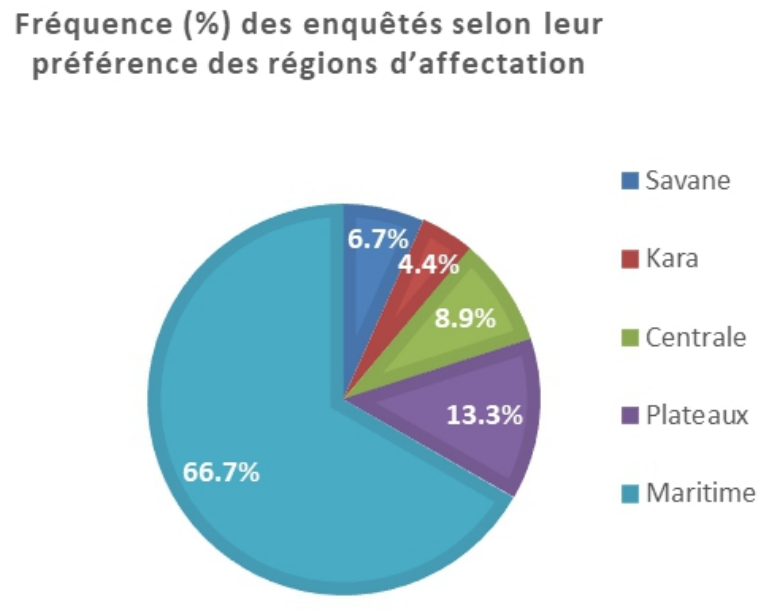

Graphique 3 : Répartition des enquêtés selon leur préférence de région d'affectation

Selon les résultats du graphique 3, les enquêtés préfèrent plus la région maritime $(66,7 \%)$, suivi de la région des plateaux $(13,3 \%)$ qui se trouve dans un rayon moyen de $150 \mathrm{~km}$ de Lomé. On comprend par ce graphique que la majorité des enquêtés préfère la région maritime qui comporte Lomé, la capitale du Togo.

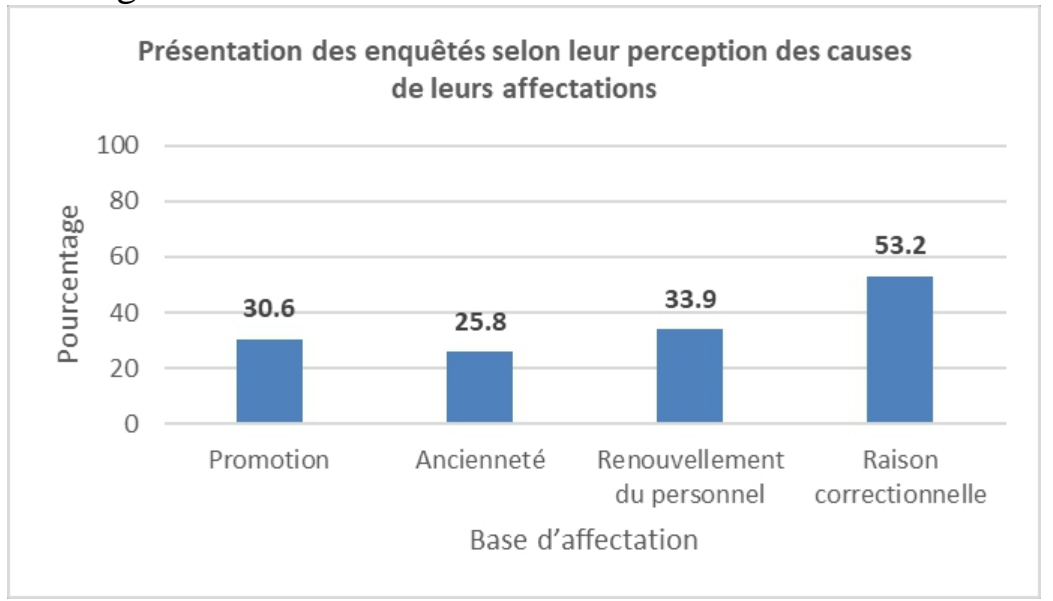

Par les résultats de ce graphique 4, on constate que 53,2\% des enquêtés lient leur affectation à une raison punitive ou correctionnelle. Ce qui démontre également la non satisfaction de l'affectation. La mobilité est perçue dans ce contexte, non pas comme un outil d'ajustement des ressources humaines, mais comme un moyen de sanction ou de pression. 


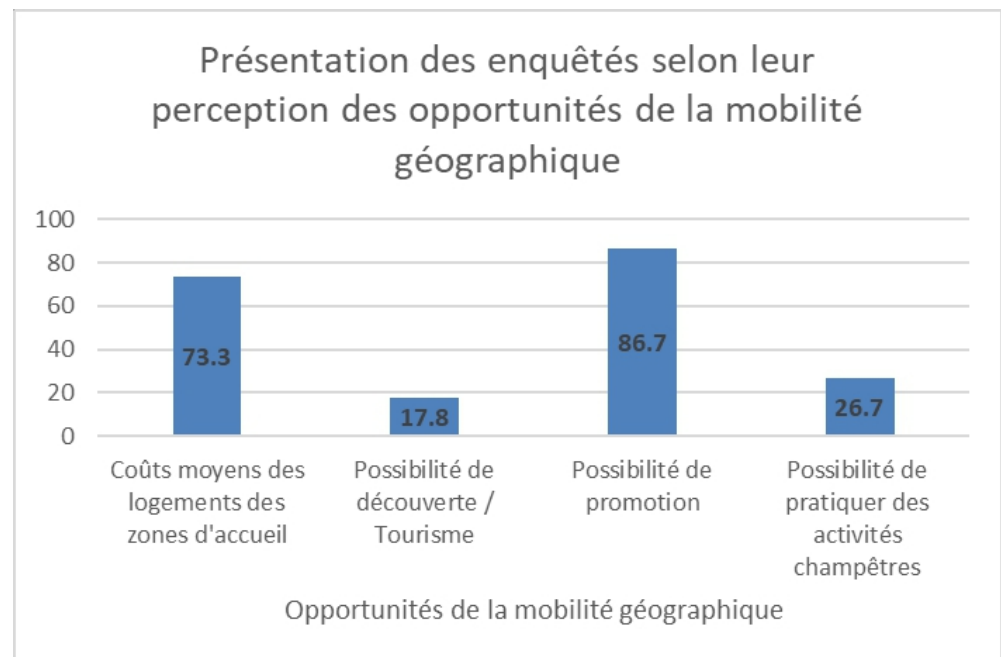

Graphique 5 : Répartition des enquêtés selon leur perception des opportunités de la mobilité professionnelle

Par ce graphique 5, on constate que $86,7 \%$ des enquêtés trouvent la possibilité d'être promu comme une opportunité liée à la mobilité géographique.

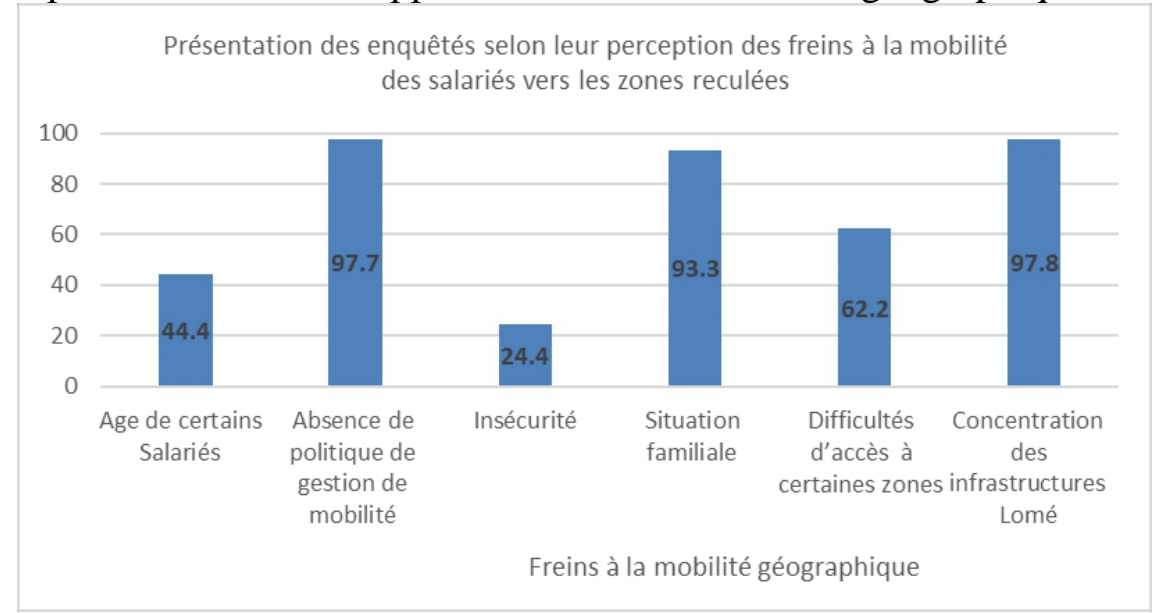

Graphique 6 : Répartition des enquêtés selon leur perception des freins à la mobilité géographique

Ce graphique montre que la concentration des infrastructures à Lomé, la capitale, constitue le premier frein à la mobilité géographique des salariés vers l'intérieur du pays. En effet, si la plupart des salariés veulent rester à Lomé, justement c'est pour profiter des infrastructures (le port, l'aéroport, les grandes écoles, les grands hôpitaux, les ministères...) qui y sont concentrées $(97,8 \%$ des enquêtés). La deuxième raison qui explique la difficulté de la mobilité géographique au Togo est liée à l'absence d'une stratégie ou d'une politique en la matière (97,7\% des enquêtés). 
(iii)

\section{Le vécu de la mobilité géographique}

Présentation en fréquence (\%) des enquêtés selon les vécus de leur mobilité géographique

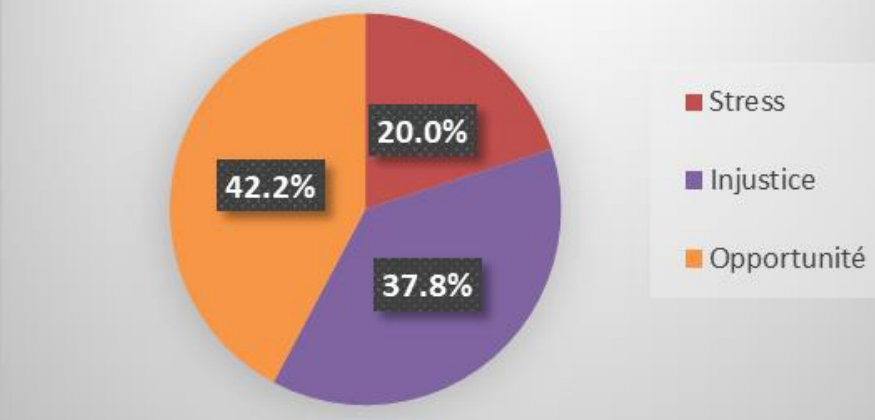

Graphique 7 : Répartition des enquêtés selon les vécus de leur mobilité géographiques

Les sentiments vécus de la mobilité géographique par les enquêtés sont divers. Ces sentiments vont du défavorable (sur les plans psychologiques et physiques) pour l'individu, au favorable. En effet, si certains enquêtés disent avoir vécu leur affectation comme une injustice (37,8\%), d'autres ont vécu des moments de stress (20\%). Par ailleurs, pour d'autres encore, le fait de changer de milieu a constitué une occasion pour eux de se développer sur le plan professionnel $(42,2 \%)$.

\section{(iv) Les propositions de solutions}

Tableau : Présentation des enquêtés selon les solutions proposées

\begin{tabular}{|l|c|c|}
\hline Propositions & Effectif & $\begin{array}{c}\text { Pourcentage } \\
(\%)\end{array}$ \\
\hline $\begin{array}{l}\text { Mettre en place une politique de gestion } \\
\text { des mobilités géographiques }\end{array}$ & 44 & 97,8 \\
\hline Mettre en place des primes d'éloignement & 42 & 93,3 \\
\hline $\begin{array}{l}\text { Tenir compte des souhaits des agents au } \\
\text { recrutement (préciser les milieux } \\
\text { d'affectation lors des recrutements) }\end{array}$ & 39 & 86,7 \\
\hline $\begin{array}{l}\text { Tenir compte des situations matrimoniales } \\
\text { des agents }\end{array}$ & 25 & 55,6 \\
\hline $\begin{array}{l}\text { Limiter officiellement la durée de } \\
\text { résidence dans un lieu d'affectation }\end{array}$ & 15 & 33,3 \\
\hline $\begin{array}{l}\text { Ne pas utiliser les affectations pour des } \\
\text { raisons punitives }\end{array}$ & 15 & 33,3 \\
\hline $\begin{array}{l}\text { Construire des infrastructures modernes } \\
\text { dans les zones reculées }\end{array}$ & 15 & 33,3 \\
\hline Spécialiser les régions & 09 & 20 \\
\hline
\end{tabular}

Le tableau ci-dessus montre que pour les enquêtés, la mise en place d'une politique de gestion de la mobilité géographique (avec des critères 
objectifs prédéfinis) constitue la principale solution. Un pourcentage de 97,8 des enquêtés adhèrent à cette proposition. Cette proposition permettrait d'éviter des mobilités hasardeuses ou punitives capables d'affecter négativement le bien être des travailleurs et la productivité des entreprises.

En somme, à travers les résultats de la présente étude, l'on peut retenir que la mobilité géographique au Togo est perçue et vécue différemment par les travailleurs affectés. On peut également constater une ambivalence dans ces perceptions et vécus. Si d'une part, la mobilité géographique apparait comme un frein pour certains travailleurs, par rapport aux problèmes qu'elle engendre (problème lié à l'âge, à la vie familiale, aux difficultés d'accès de certaines zones, etc.), d'autre part, elle constitue une occasion pleine d'opportunités (promotion, découverte d'autres régions, etc.). En outre, la mobilité géographique engendre comme vécu le stress, le sentiment d'injustice chez certains travailleurs et l'épanouissement chez d'autres.

\subsection{Discussion}

L'objectif de la présente étude est d'analyser les perceptions et les vécus de la mobilité géographique par les agents affectés. A l'issue des résultats obtenus, on constate que les enquêtés ont une perception mitigée de la mobilité géographique. $\mathrm{Si}$, certains enquêtés déclarent que leur affectation a souvent répondu à une exigence de leur carrière qui est la promotion (perception positive), d'autres (majoritaires) la trouvent comme injuste et/ou une source de stress, donc perception négative (Cf. graphique 3 et tableau 8). Les résultats obtenus montrent également que la mobilité géographique pensée et utilisée comme un outil de management des ressources humaines devrait correspondre à une intention du travailleur.

En effet, l'intention est fondée sur une double conditionnalité : d'une part, l'espérance d'un gain (comme dans le cas d'une perception positive, qui correspond à l'attente d'une promotion) et d'autre part, la volonté de se conformer à un contrat psychologique (Rousseau, 1989). Dans le cas du contrat psychologique, le non-respect constitue une violation des droits de l'une ou de l'autre des parties. Dans ce contexte de mobilité géographique, c'est l'employé (travailleur) qui pense au non-respect dont les conséquences sont le sentiment d'injustice et le stress. Ce qui va dans le même sens que les conclusions de Hellemans et Equeter (2016), selon lesquelles la mobilité géographique peut être subie (avec parfois comme conséquence le stress) ou volontaire.

Par ailleurs, si la motilité ou mobilité désigne l'aptitude psychologique, culturelle, physique aussi à se mouvoir ( Beaucire \& Desjardins, 2015), on peut constater chez certaines une faible ou une forte aptitude dans ces différentes dimensions. C'est ainsi qu'à travers le graphique 3 , on constate que la majorité des salariés n'est pas satisfaite de son nouveau 
lieu de travail. L'insatisfaction des travailleurs s'explique par les freins organisationnels auxquels font face les salariés affectés vers les zones reculées. Ces freins sont relatifs également à l'absence de politique de mobilité, l'absence de prime d'éloignement, l'absence de logement dans certaines zones, le manque surtout d'infrastructures sociales de base, la difficulté d'accès au lieu de travail, l'affectation correctionnelle (punitive), l'absence d'opportunité d'avoir ou d'initier des projets sources de revenu complémentaire.

Les résultats de la présente étude vont également dans le sens de ceux de Brutel, Jegou \& Rieu (2000) qui précisent que la mobilité géographique est toujours favorable à la promotion professionnelle, et vice versa. En effet, les résultats révèlent que l'une des raisons de la mobilité géographique au Togo est également la promotion professionnelle.

Cette étude corrobore celle de Bonnet, Collet et Maurines (2006), qui a mis en évidence une interdépendance entre la carrière familiale et la mobilité géographique des couples, même si elle ne permet pas d'appréhender les ajustements et les négociations que les couples effectuent afin de garantir le rapprochement conjugal. Ainsi, les résultats du tableau 7 montrent que des salariés enquêtés perçoivent que leur situation familiale représente un frein à leur mobilité géographique.

Cette étude exploratoire a également le mérite de montrer que la mobilité géographique présente des opportunités pour les salariés : les coûts moyens des logements des zones d'accueil (par rapport à la cherté de la vie des zones de départ), la possibilité de pratiquer des activités champêtres, sans oublier la possibilité de découverte ou de tourisme.

D'une manière générale, l'objectif de cette étude est atteint dans la mesure où elle a permis de déterminer les perceptions et les vécus des enquêtés par rapport à la mobilité géographique au Togo. Ce qui permettra de prendre des dispositions dans le cadre de la réduction des disparités entre zones. Cela nous renvoie à deux implications, théorique et pratique.

Sur le plan théorique, elle renforce la compréhension de la théorie du contrat psychologique et ses implications (Rossano, Abord de Chatillon, Desmarais, 2015) en montrant les conséquences de la rupture du contrat psychologique par les perceptions et le vécu des travailleurs enquêtés. En effet, l'affectation confronte le travailleur à différentes facteurs (la séparation du milieu habituel ou de la famille et l'adaptation à un nouvel environnement, etc.) pouvant affecter son bien-être. Sur le plan pratique, la présente étude permet d'opérationnaliser la théorie de l'action raisonnée de Ajzen (1991) en indiquant que, la mobilité géographique comme un véritable outil (efficace) de management des ressources humaines, doit préparer les travailleurs au travers du jeu de proposition des «intentions ». Ainsi, le fait, avant une mutation, qu'on donne l'occasion au travailleur de faire plusieurs choix, 
prépare ce dernier à l'acceptation du nouveau lieu d'affectation. C'est ce qu'on nomme la «préparation psychologique au consentement». C'est l'une des conditions qui facilite l'intégration $\mathrm{du}$ travailleur dans son nouvel environnement de travail et par conséquent garantit sa productivité au poste.

Cependant, il faut déplorer la représentativité des catégories de travailleurs concernés par le phénomène au Togo qui réduit ainsi le niveau de généralisation des résultats.

\section{Conclusion}

La mobilité géographique évoque un déplacement d'une zone à une autre. Dans un premier temps, elle peut bien entendu, être facilitée - ou freinée - par des caractéristiques en termes d'infrastructures (transport, sanitaire, hôtelier, universitaire, etc.). Ces freins peuvent être intériorisés et se présenter sous la forme cognitive ou psychologique.

En effet, les résultats obtenus montrent que l'absence de politique de mobilité, l'absence de prime d'éloignement, l'absence de logement dans certaines zones, le manque d'infrastructures, la difficulté d'accès au lieu de travail, l'affectation correctionnelle (punitive) constituent les freins à la mobilité géographique tandis que les coûts moyens des logements des zones d'accueil, la cherté de la vie des zones de départ, la possibilité de pratiquer des activités champêtres et la possibilité de découverte ou de tourisme représentent les opportunités (mesures incitatives). Au-delà de ces perceptions, il faut également noter qu'elles entraînent malheureusement des conséquences négatives comme les sentiments d'injustice et le vécu du stress. Dans le cadre de l'amélioration de la mobilité géographique, la littérature suggère deux principes d'action : inciter les agents au mouvement en créant des dispositifs attractifs d'une part et faire respecter leur engagement à être mobiles d'autre part.

La présente étude est d'un grand intérêt pour les managers. Elle a permis d'identifier les opportunités et de proposer des solutions incitatives à la mobilité géographique des travailleurs. Toutefois, il faut relever des limites méthodologiques relatives à l'échantillon d'étude relativement faible pour permettre des croisements statistiques. Il s'avère donc important de continuer des études dans ce champ de la mobilité professionnelle en mettant en lien par exemple la perception de la mobilité géographique et le bien-être au travail des salariés. Ce qui permettra d'évaluer l'ampleur des conséquences négatives de la mobilité géographique surtout non consentie sur les ressources humaines.

\section{References :}

1. Adedeji, S. O. et Olaniyan, O. (2011). L'amélioration des conditions des enseignants et de l'enseignement en milieu rural en Afrique, UNESCO-IICBA, Addis Abéba. 
2. Ajzen, I. (1991). The theory of planned Behavior. Organizational Behavior et Human decision Processes, 50(2), 179-212.

3. Chitou, I. (2013). Éthique et pratique managériale dans les entreprises publiques en Afrique subsaharienne : pourquoi tant de difficultés ? Le cas du Togo. Gestion et management public, volume 1/4(2), 23-35. doi:10.3917/gmp.004.0023.

4. Beaucire F., Desjardins X. (2015). Notion d'urbanisme par l'usage, Paris, Publications de la Sorbonne, 120 pages.

5. Bernela, B. (2017). Trajectoires professionnelles et géographiques : l'étude de trois générations de docteurs. Formation emploi [En ligne], mis en ligne le 15 octobre 2019, consulté le18 février 2020. URL : http://journals.openedition.org/formationemploi/5154

6. Blum, A., De La gorce, G. et Thélot, C. (1985), Mobilité sociale et migration géographique. Population, p. 397-434.

7. Bonnet, E, Collet, B. et Maurines, B. (2006). Carrière familiale et mobilité géographique professionnelle. Cahiers du Genre, 2 (41), 7-98

8. Brutel, C., Jegou M. et Rieu C. (2000). La mobilité géographique et la promotion professionnelle des salariés : une analyse par aire urbaine. In Economie et statistique, 336(1), 53-68.

9. Deschênes, A., Beaudry, C., Laflamme, J. \& Aguir, M. (2018). La mesure de l'attraction dans les organisations situées en région périphérique : vers un modèle de l'attraction régionale des travailleurs du savoir. La Revue des Sciences de Gestion, 291-292(3), 13-22. doi:10.3917/rsg.291.0013.

10. Dumartin, S. (1995). Mobilité géographique et insertion professionnelle des jeunes. In: Economie et statistique, $\mathrm{N}^{\circ} 283-284$.

11. Frank, O. et Snijders, T. (1994). Estimating the size of hidden populations using snowball sampling. Journal of official stattistics, 10 , $53-67$.

12. Hellemans, C. et Equeter, E. (2016). Mobilité professionnelle. In G. Valléry, M.-E. B.

13. Iacono, G. (2008). Gestion des ressources humaines : Cinq défis pour l'avenir. Collection : Paris.

14. Johnston, L. G. et Sabin, K. (2010). Sampling hard-to-reach populations with respondent driven sampling. Methodological innovations online, $5(2), 38-48$

15. Kazimna, P. (2012). Représentation du secteur privé et motivation des salariés togolais, Doctorat de Psychologie du Travail et des Organisations, Université de Lomé.

17. Maclouf, E. \& Wierzbicki B. (2008). La mobilité géographique pour optimiser la gestion des ressources humaines publiques ? Politiques et management public. 26 (2), 53- 74 
16. 17. Mignonac, K. (2004). Comprendre et favoriser la disposition des ingénieurs et cadres envers la mobilité géographique intraorganisationnelle, La gestion des carrières. Enjeux et perspectives. Dunod : Paris.

18. Mokounkolo, R. (2019). Introduction. In R. Mokounkolo, Ngueutsa, R. Courcy, F. Ntsame Sima, M., et Achi, N. (Eds.), Les pays du sud face aux défis du travail (p. 7-14). L'Harmattan : Paris.

19. Pari, P. (2008). Représentation de la fonction publique et motivation au travail : cas des agents du secteur public de Lomé, Doctorat de Psychologie du Travail et des Organisations, Université de Lomé.

20. République Togolaise. (2018). Plan National de Développement 2018-2022, Togo. Retrieved from https://www.republiquetogolaise.com/pnd.

21. Rossano, M., Abord de Chatillon E., Desmarais C. (2015). Rupture du contrat psychologique et risques psycho-sociaux : une recherche intervention dans le cadre de la théorie de la conservation des ressources. Revue de gestion des ressources humaines, $\mathrm{N}^{\circ} 95,58-77$.

22. Rousseau, D. M. (1989). Psychological and implied contracts in organizations. Employee responsibilities and rights journal, 2(2), 121139.

23. Vandersmissen, M. H., Villeneuve, P. et Thériault, M. (2001/4). L'Espace géographique, 97-110. 